\title{
Comparison of HIV-1 viral loads, CD4-Th praziquantel treatment among adults infected or uninfected with Schistosoma mansoni in fishing villages of north-western Tanzania
}

\author{
HUMPHREY D. MAZIGO ${ }^{1,2^{*}}$, DAVID W. DUNNE³, DOMENICA MORONA ${ }^{1}$, SAFARI M. KINUNG'HI ${ }^{4}$ and FRED \\ NUWAHA ${ }^{2}$ \\ 'Department of Medical Parasitology and Entomology, School of Medicine, Catholic University of Health and \\ Allied Sciences, P.O. Box 1464, Mwanza, Tanzania \\ ${ }^{2}$ School of Public Health, College of Health Sciences, Makerere University, Kampala, Uganda \\ ${ }^{3}$ Division of Microbiology \& Parasitology, Cambridge University, Cambridge, United Kingdom \\ ${ }^{4}$ National Institute for Medical Research, Mwanza Research Centre, Mwanza, Tanzania
}

\begin{abstract}
Background: It is hypothesised that $\mathrm{Th}_{2}$ immunological environment associated with Schistosoma mansoni infection might favour replication of HIV-1 in co-infected individuals, results in increased viral loads. On the other hand, deworming using praziquantel might result in reduction of HIV-1 viral loads and increased CD4 ${ }^{+}$ cell counts. This study was therefore, carried out to compare HIV -1 plasma loads, CD4-Th2-lymphocytes and the effects of praziquantel treatment on HIV-1 plasma loads and CD4+ cell counts among HIV-1 seropositive individuals infected or uninfected with S. mansoni.

Methodology: A 9-month prospective longitudinal study was conducted among HIV-1 infected individuals aged 21-55 years with $\mathrm{CD}_{4}{ }^{+}$cell counts $\geq 350$ cells $/ \mu \mathrm{L}$ in fishing villages of North-Western Tanzania. Single stool samples were examined for S. mansoni eggs using Kato Katz technique at 6-month follow-up and 12 weeks after treatment. Venous blood samples were collected at baseline, at three and six-month follow-up and 12 weeks after praziquantel treatment for HIV-1 plasma viral loads and CD4 ${ }^{+}$cell quantification.

Results: Of the 50 HIV-1 infected participants at baseline, $44 \%(22 / 50,95 \% \mathrm{Cl} ; 30.58-58.35)$ were found to be co-infected with S. mansoni at 6-month follow-up with a mean of 93.26GM-epg (95\% Cl: 60.42-143.95). The median $\mathrm{CD}_{4}{ }^{+}$cell counts did not differ significantly between individuals infected with HIV-1 and those coinfected with HIV-1 and S. mansoni at baseline $(P=0.62)$, 3-month $(P=0.64)$ and 6-month $(P=0.41)$ follow-up. Monthly decrease in $\mathrm{CD} 4^{+}$cells did not differ significantly between the two groups at all follow-up points (30.39cell $/ \mu \mathrm{L}$ versus -31.35 cells $/ \mu \mathrm{L}, P=0.89$ ). Those infected with $S$. mansoni had a significantly higher mean $\log _{10}$ HIV-1 plasma viral load at baseline $(5.98 \pm 3.06$ versus $9.21 \pm 1.91$ copies $/ \mathrm{ml}, P<0.0001)$ and 3 -month follow-up ( $8.19 \pm 2.17$ versus $9.44 \pm 1.99$ copies $/ \mathrm{ml}, P<0.042$ ) compared to those infected with HIV-1 only. This difference was not evident at the time of S. mansoni diagnosis at 6-month time point. Praziquantel treatment in co-infected individuals $(n=12)$ did not result in any change in $\mathrm{CD}_{4}{ }^{+}$cell counts and mean HIV-1 plasma viral loads $(\mathrm{t}=-0.9156, P=0.38)$, comparing baseline and 3 -month follow-up after treatment. No correlation was observed between log S. mansoni egg counts and $\log _{10}$ HIV-1 RNA viral loads ( $r=-0.066$, $P=0.77)$ at six-month follow-up in co-infected individuals $(n=22)$.

Conclusion: HIV-1 plasma viral loads varied significantly among mono and co-infected individuals at baseline and 3-month follow-up. However, $\mathrm{CD}_{4}{ }^{+}$cell counts did not vary between the two groups at all follow-up time points. Praziquantel treatment of co-infected individuals did not result in changes in $\mathrm{CD}_{4}{ }^{+}$cell counts and HIV-1 plasma viral loads.
\end{abstract}

Keywords: HIV-1, Schistosoma mansoni, co-infection, HIV loads, praziquantel, Tanzania

\section{Introduction}

Immunological evidence from laboratory animal models and human host(s) (Secor, 2005, Secor, 2006, Secor, 2012) indicate that helminth infections may cause immune modulation which may increase the susceptibility of infected individuals to other infections (Mwinzi et al., 2001, Secor et

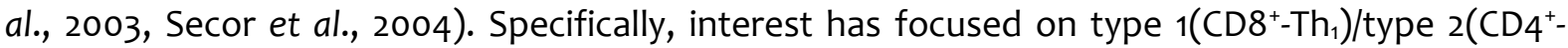
$\mathrm{Th}_{2}$ ) arms of the immune system, in which, type $1\left(\mathrm{Th}_{1}\right)$ immune response, which secretes interferon- $\gamma$, interleukin-2 (IL-2), promotes phygocytosis and intracellular killing of viral particles, including HIV-1 (Clerici \& Shearer, 1993; Bentwich et al., 1995). Helminths parasites such as

\footnotetext{
*Correspondence E-mail: humphreymazigo@gmail.com
} 
Schistosoma mansoni induce type 2 immune responses characterized by the release of interleukin (IL)-4, IL-5, IL-10 and IL-13, which stimulate the proliferation of B-lymphocytes cells, antibodies and eosinophils production (Clerici \& Shearer, 1993; Mwinzi et al., 2001; Secor et al., 2004; Borkow \& Bentwich, 2004). The ability of helminth parasites to modulate immune response, which shifts from T-helper-1 to predominant T-helper-2 cytokines, is thought to impair the type-1 response (Sher \& Coffman, 1992; Maizels et al., 1993; Kalinkovich et al., 1998; Maizels \& Yazdanbakhsh, 2003), which, in turn (Clerici \& Shearer, 1993), increases the susceptibility of $\mathrm{Th}_{0}$ and $\mathrm{Th}_{2}$ cells to HIV-1 infection (Sher \& Coffman, 1992; Clerici \& Shearer, 1993; Secor, 2005). This observation on immune dysregulation has led to the hypothesis that immune modulations resulting from chronic S. mansoni infection are associated with HIV-1 disease progression, through increased HIV-1 replication and decrease $\mathrm{CD}_{4}^{+}$levels (Bentwich et al., 2000; Secor et al., 2003; Borkow \& Bentwich, 2004; Secor, 2006, 2012; Mazigo et al., 2013).

In addition, in vitro studies have shown that $\mathrm{CD}_{4}{ }^{+}-\mathrm{Th}_{2}$ lymphocytes cells from $\mathrm{S}$. mansoni infected individuals express chemokines receptors (CCR5 and CXCR4) which render these cells more susceptible to HIV-1 infections, as compared to the cells of helminth free individuals (Kalinkovich et al., 2001; Secor et al., 2003; Secor, 2012). Alternatively, in S. mansoni endemic areas, where individuals are infected by the parasite at an early age, their $\mathrm{CD} 4^{+} \mathrm{T}$-lymphocytes are stimulated by the parasites' antigens and, by the time they are infected by HIV-1 at older ages, they may end up with higher viral loads and progress very fast to AIDS stages (as measured in term of $\mathrm{CD}^{+}$cell counts) (Bentwich et al., 1999; Borkow \& Bentwich, 2004; Secor, 2012). If these assumptions are correct, it is expected that individuals co-infected with S. mansoni+HIV-1 with a heavy intensity of S. mansoni infection will have higher HIV-1 viral loads as compared to HIV-1 mono-infected individuals. However, up to date, inconsistent results have emerged (Lawn et al., 2000, Brown et al., 2004, Kallestrup et al., 2005a). Thus, the role of immune modulation of $T h_{1} / T_{2}$ responses associated with $\mathrm{S}$. mansoni on the HIV-1 pathogenesis remains a subject for further studies, especially in sub-Saharan Africa.

The observations on immune dysregulation as well as chronic immune activation associated with HIV-1+ S. mansoni in co-infected individuals, it has been argued that early treatment of S. mansoni using praziquantel (PZQ) could reduce the rate of viral replications (Erikstrup et al., 2008), improve prognosis and delay the initiation of antiretroviral treatment (ART) (Walson \& John-Stewart, 2008; Modjarrad \& Vermund, 2010). In fact, PZQ treatment reduces the density of chemokines receptors (CCR5 and CXCR4), which, in turn, reduce/control the replication of HIV-1 (Secor et al., 2003). The reported evidence has remained inconsistent, increased (Lawn et al., 2000; Brown et al., 2005) or decreased (Brown et al., 2005) HIV-1 RNA viral loads have been documented after PZQ treatment in S. mansoni+HIV-1 co-infected individuals. In addition, few community-based studies have addressed this issue in HIV-1 cohorts constantly exposed to a high risk of S. mansoni transmission and not qualifying for initiation of ART (Lawn et al., 2000; Kallestrup et al., 2005a).

In the current study, we hypothesized that, if $\mathrm{Th}_{2}$ immunological environment associated with S. mansoni favours the replication of HIV-1 in co-infected individuals, then individuals who are infected with $\mathrm{S}$. mansoni prior to be infected by HIV-1, will, by the time they acquire HIV-1 infection the disease, progress faster (as measured by the decrease in $\mathrm{CD}_{4}{ }^{+}$cell counts and the increase in HIV-1 viral load) overtime than those mono-infected with HIV-1. Furthermore, PZQ treatment in co-infected individuals will result into a decrease in plasma HIV-1 viral loads. Thus, the present study was therefore conducted to compare (a) Human Immunodeficiency Virus-1 plasma loads, CD4-Th2-lymphocytes and (b) effects of praziquantel treatment on HIV-1 plasma loads and CD4 ${ }^{+}$ cell counts among HIV-1 seropositive individuals infected or uninfected with S. mansoni.

\section{Materials and Methods}

\section{Study area, population and inclusion criteria}


Descriptions of the study area, inclusion and exclusion criteria and sampling procedures of the study participants are described elsewhere(Mazigo et al., 2014). Briefly, the study was conducted at Ilemela district, located on the southern shore of the Lake Victoria. The study included in habitants living at Kayenze, Igalagala, Sangabuye and Igombe villages. The villages were chosen for the study because of their close proximate to the lake and high prevalence of Schistosoma mansoni (Mazigo et al., 2014). The inhabitants of the study villages depend on the Lake for domestic and economic activities such as washing, bathing, cooking, drinking and recreation (Malenganisho et al., 2008).

All people who had lived in the study villages for more than two years (referred as permanent residence) and were aged 21 to 55 years were eligible for enrolment. Individuals with a history of treatment for schistosomiasis (praziquantel) in the past six months and those who were on antiretroviral treatment (ART) were excluded from the study at the baseline. A two-step sampling procedure was used to select households and household members to participate in the study (Mazigo et al., 2014).

\section{Study design and follow-up}

This was a prospective longitudinal study in which a cohort of HIV-1 infected individuals with CD4 ${ }^{+}$ cell counts $\geq 350$ cells/ $\mu \mathrm{L}$ was followed for nine (9) months. Study participants were followed at three and six months to collect venous blood samples for monitoring of CD4 $4^{+} \mathrm{T}$-lymphocytes and HIV-1 plasma viral loads. Fifty study participants completed a 6-month follow-up and were screened for S. mansoni infection. Those who had S. mansoni eggs in their stool samples were treated using Praziquantel (40mg/kg) and followed up at 12 weeks after treatment to assess the efficacy of treatment and its effects on HIV-1 plasma viral loads. Twelve weeks after treatment, only 12 study participants who had detectable S. mansoni eggs in their stool samples were available for assessment. The main reasons for loss to follow-up were migration, refusal to continue to participate and absence from home.

HIV-1 viral loads and $\mathrm{CD}_{4}{ }^{+}$cell counts were compared in individuals who were co-infected with S. mansoni+HIV-1 and those who were HIV-1 mono-infected at six-month follow-up. The same outcomes were compared among the co-infected individuals before and after praziquantel treatment. Furthermore, we assessed if the intensity of S. mansoni infection correlated with HIV-1 RNA viral loads.

\section{Screening for HIV-1 infection, $\mathrm{CD}_{4}^{+}$analysis and plasma viral load quantification}

Demographic information on sex, age, marital status and other socioeconomic information were collected by questionnaire. Human Immunodeficiency Virus-1 testing was conducted according to the Tanzanian National HIV algorithms which recommend the use of a rapid test qualitative immunoassay (Lyamuya et al., 2009). The quantification of $\mathrm{CD}^{+}$cells was carried out using FACSCalibur machine (Becton Dickinson-BD Biosciences, San Jose, CA, USA) following standard procedures (Borato et al., 2012).

Plasma samples for HIV-1 RNA viral load quantification were collected and stored at $-80^{\circ} \mathrm{C}$ within 24 hours of blood collection. Plasma HIV-1 viral loads in all samples were determined by Artus $^{\circledR}$ HI Virus-1 RG TR-PCR kit (Qiagen GmbH, Qiagen strasse I, D-40724 Hilden) following the manufacturer's instructions. The diagnostic test kit utilizes the reverse transcription polymerase chain reaction (RT-PCR). The lowest analytical detection limit in consideration of the purification of the artus HI Virus-1 RG RT-PCR Kit in combination with the Rotor-Gene Machine is 35.8 log RNA copies $/ \mathrm{ml}$.

\section{Parasitological screening for Schistosoma mansoni and treatment}

A single stool sample was collected from all study participants at six and 12 weeks after treatment, from which, four (4) Kato Katz thick smears were prepared from different parts of the single stool sample using a template of $41.7 \mathrm{mg}$ (Vestergaard Frandsen, Lausanne, Switzerland), following a 
standard protocol (Katz et al., 1972). For quality assurance, a random sample of 10\% of the negative and positive Kato Katz thick smears were re-examined by a third technician.

After examination at six months of follow-up, all individuals found infected with S. mansoni were treated using a single dose of praziquantel $(40 \mathrm{mg} / \mathrm{kg})$. Treatment was performed under the direct observation (DOT) of a qualified a nurse. Study participants received a cup of tea and half a bread before treatment to relieve any potential side effects of praziquantel. In addition, after treatment, participants were requested to remain at the treatment point for two hours for observation by a medical doctor.

\section{Ethical Considerations}

Ethical approval was obtained from the Higher Degrees Research and Ethics Committee of the School of Public Health, Makerere University (Institutional Review Board, Ref: 00005856/2011) and from the Bugando University College of Health and Allied Sciences-Institutional Review Board, (BREC/001/32/2011). Ethical clearance was granted by the National Ethical Review Committee, National Institute for Medical Research, Tanzania and the study was registered in the clinical trial network, Clinical Trial (Number: NCT-01541631).

\section{Data analysis}

The data were double-entered using CSPro and the final data set was stored in a MySQL database. Data analysis was performed using Stata version 13 (Stata Corp, College station, Texas, USA). The Mann-Whitney test was used to compare median $\mathrm{CD}_{4}^{+}$cell counts between those with and without S. mansoni infection at baseline, three and six month's follow-up. Plasma HIV-1 viral loads were $\log \left(\log _{10}\right)$ transformed to approximate a normal distribution. The mean $\log _{10}$ plasma HIV-1 RNA viral loads were compared between individuals who were co-infected and monoinfected using t-test for continuous outcomes.

At baseline and 3-month follow-up, samples from 19 individuals were detected to be below the detectable range of the machine and were classified as having 34copies $/ \mathrm{ml}$ for the purpose of analysis. Monthly changes in $\mathrm{CD}_{4}{ }^{+}$cell counts and HIV-1 viral loads were analysed using a mixed linear model for repeated measures. The effect of PZQ treatment was evaluated by comparing change in $\log _{10}$ plasma HIV-1 RNA viral load between baseline and 12 weeks (three months) after treatment. The analysis of follow-up viral loads after treatment mainly focused on comparing those remained with detectable S. mansoni eggs and those who had no detectable eggs at 12 weeks after treatment.

The arithmetic mean of S. mansoni egg counts for each participant was calculated from the counts of four Kato Katz thick smears and multiplied by 24 to obtain individuals eggs per gram of faeces. S. mansoni egg counts were over-dispersed so were logarithmically transformed and used to calculate the geometric mean egg per gram of faeces (GM-epg) that was obtained as the antilog of the mean of the transformed egg counts. Pearson's and Spearman's correlation coefficient for individuals co-infected with S. mansoni and HIV-1 was used to determine any correlation between HIV plasma loads and S. mansoni intensity of infection. Statistical significance was set at $P \leq 0.05$.

\section{Results}

\section{Characteristics of study participants}

At baseline, a total of 2,138 individuals were recruited and enrolled for HIV-1 screening. Of these individuals, 167 were identified as HIV-1 positive at baseline, 77 had CD4 $4^{+}$T-lymphocytes counts $<350$ cells $/ \mu \mathrm{L}$ and 90 had $\mathrm{CD} 4^{+}$T-lymphocytes counts $\geq 350$ cells $/ \mu \mathrm{L}$. The fifty individuals who completed a six-month follow-up were aged 21-55 years, the median age was 34.5years (IQR=2843 years). The two groups of the study participants, those mono-infected with HIV-1 and those co-infected with HIV-1 and S. mansoni, did not differ by sex distribution $\left(X^{2}=0.1523, P=0.69\right)$ and 
age $(t=0.9193, P=0.36)$. The majority of the study participants were from Sangabuye $(44 \%)$ and Kayenze villages. Baseline $\mathrm{CD} 4{ }^{+}$cell counts and demographic information were compared for the study participants who were present and absent at 6 months and 9 months of follow-up. There was no significant difference on age $(t=-1.6990, P=0.09)$. There was a difference is sex distribution $\left(X^{2}=5.9533, P<0.015\right)$, village of residence $\left(X^{2}=7.4772, P<0.001\right)$, mean $C D 4^{+}$cell counts $(\mathrm{t}=-6.5360, P<0.0001)$, whereby those who were present had a higher mean $\mathrm{CD} 4^{+}$than those who were absent $(716.14 \pm 320.25$ versus $395.97 \pm 273.36$ cells $/ \mu \mathrm{L}$ ) (Table 1$)$.

Table 1: Comparison of $\mathrm{CD}_{4}^{+}$levels and HIV-1 plasma viral loads among participants infected with HIV-1 only and those co-infected with HIV-1 and S. mansoni at the 6 month follow up time point

\begin{tabular}{|c|c|c|c|c|}
\hline Variables & HIV-1 only $(n=22)$ & $\begin{array}{l}\text { HIV-1 + S. mansoni } \\
\text { co-infected }(n=28)\end{array}$ & F-test & P-values \\
\hline \multicolumn{5}{|c|}{ CD4 ${ }^{+}$- T-lymphocytes (Median and Interquartile range) } \\
\hline Baseline & $\begin{array}{c}574 \\
(I Q R: 484.5-801.0)\end{array}$ & $\begin{array}{c}654.5 \\
(I Q R: 546.0-773.0)\end{array}$ & ---- & 0.62 \\
\hline 3 month & $\begin{array}{c}514.5 \\
\text { (IQR: } 430.5-723.0)\end{array}$ & $\begin{array}{c}615 \\
(\mathrm{IQR}: 462.0-718.0)\end{array}$ & ---- & 0.64 \\
\hline 6 month & $\begin{array}{c}470.5 \\
(I Q R: 385.5-593.0)\end{array}$ & $\begin{array}{c}567 \\
(I Q R: 412.0-614.0)\end{array}$ & ---- & 0.41 \\
\hline \multicolumn{5}{|c|}{ HIV-1 viral load (Mean log10 RNA copies/ml of plasma) } \\
\hline Baseline & $\begin{array}{l}5.98 \pm 3.06 \\
(95 \% \mathrm{Cl}: 4.79-7.17)\end{array}$ & $\begin{array}{l}9.21 \pm 1.91 \\
(95 \% \mathrm{Cl}: 8.36-10.06)\end{array}$ & $t=-4.72553$ & 0.0001 \\
\hline 3 month & $\begin{array}{l}8.19 \pm 2.17 \\
(95 \% \mathrm{Cl}: 7.35-9.03)\end{array}$ & $\begin{array}{l}9.44 \pm 1.99 \\
(95 \% \mathrm{Cl}: 8.55-10.32)\end{array}$ & $t=-2.0892$ & 0.042 \\
\hline 6 month & $\begin{array}{l}10.49 \pm 1.29 \\
(95 \% \mathrm{Cl}: 9.99-10.99)\end{array}$ & $\begin{array}{l}10.59 \pm 1.44 \\
(95 \% \mathrm{Cl}: 9.96-11.24)\end{array}$ & $t=-0.2714$ & 0.79 \\
\hline
\end{tabular}

\section{Prevalence and intensity of Schistosoma mansoni}

Of all the 50 study participants who were infected with HIV-1 and had CD4 $4^{+} \geq 350$ cells/ $\mu \mathrm{L}, 44 \%$ (95\% Cl: 30.58-58.35) were co-infected with S. mansoni. Of these, $46.43 \%(n=13 / 28)$ were females and $40.91 \%(n=9 / 22)$ were males. The overall geometrical mean eggs per gram (GM-epg) of faeces of S. mansoni were 93.26GM-epg (95\%Cl: 60.42-143.95). By sex, males had a higher GM-epg, 148.41(95\% Cl: 45.19-487.28) than females, 68.41GM-epg (95\%Cl: 35.08-133.43) $(P<0.003)$.

\section{$\mathrm{CD} 4^{+} \mathrm{T}$-lymphocytes and HIV-1 plasma viral loads at baseline and follow-up}

At baseline and at the subsequent follow-up at three and six-month time point, the median $\mathrm{CD} 4^{+}$ cell counts did not differ significantly between the two groups (Table 1). There was a trend in the decrease of $\mathrm{CD}_{4}^{+}$cell counts with time, baseline to three months $(P<0.0001$, paired $t$-test), baseline to six months $(P<0.0001)$ and baseline to 12 weeks after treatment $(P<0.0147)$, for all the study participants. The mean monthly decrease in $\mathrm{CD}_{4}^{+}$cell counts from baseline to the sixmonth follow-up time point for all study participants was -30.81 cells $/ \mu \mathrm{L}(95 \% \mathrm{Cl}:-38.06$ to -23.55$)$. In relation to the $S$. mansoni infection status, those infected with HIV-1 only had a monthly decrease in $\mathrm{CD}^{+}$cell counts of -30.39 cells $/ \mu \mathrm{L}(95 \% \mathrm{Cl}$ : -40.08 to -20.69$)$ and those co-infected with HIV-1+S.mansoni had a monthly decrease of -31.35 cells/ $\mu \mathrm{L}(-42.29$ to -20.41$)$. The difference in monthly decrease of $\mathrm{CD}_{4}^{+}$counts between the two groups was not statistically significant $(P=0.89)$.

For the HIV loads, at baseline $(P<0.0001)$ and at three months $(P<0.042)$, the mean $\log _{10}$ HIV-1 plasma viral load was higher in the $S$. mansoni infected groups than the $S$. mansoni noninfected group (Table 1). However, at six-month follow-up time point, the difference in mean $\log _{10}$ HIV-1 viral load did not differ between the S. mansoni infected groups and the HIV-1 mono- 
infected $(P=0.79)$. There was a trend of increase in mean $\log _{10}$ HIV-1 plasma viral loads with time for all study participants from baseline to three months $(P<0.01$, paired $t$-test), baseline to six months $(P<0.0001$, paired $t$-test) follow -up time points and from baseline to 12 weeks after treatment $(P<0.001$, paired $t$-test). The monthly increase in log plasma HIV load for the whole study population was 0.52 copies $/ \mathrm{ml}(95 \% \mathrm{Cl}: 0.39-0.65)$ for the six-month follow-up point. In relation to S. mansoni infection, those participants who were mono-infected with HIV-1 had a significantly higher monthly increase in plasma HIV-1 load of $0.75 \mathrm{copies} / \mathrm{ml}(95 \% \mathrm{Cl}: 0.59-0.91)$ than those co-infected with HIV-1+S.mansoni, 0.23copies $/ \mathrm{ml}(95 \% \mathrm{Cl}: 0.049-0.41)$. The difference observed between the two groups in monthly increase in plasma HIV-1 loads was significant $(P<0.0001)$.

\section{Effects of treatment of S. mansoni on HIV-1 plasma viral load}

The HIV-1 plasma viral load results, both at baseline and 12 weeks after praziquantel treatment were available for 12 individuals who were co-infected at the six-month time point. Of the 22 study participants screened for S. mansoni and treated with PZQ at six-month time point, 10 were lost to follow-up due to migration from the study villages. The remaining 12 individuals, at baseline, had a mean HIV-1 plasma viral load of $8.54 \pm 1.64$ RNA copies/ml which insignificantly increased to $9.41 \pm 2.98 \mathrm{RNA}$ copies $/ \mathrm{ml}(\mathrm{t}=-0.9156, \mathrm{P}=0.38) 12$ weeks after PZQ treatment. Of the 12 individuals screened for $S$. mansoni infection at 12 weeks after PZQ, 8 had no detectable $S$. mansoni eggs in their stool samples and 4 remained with detectable $S$. mansoni eggs. At baseline, the 8 participants had a median $\mathrm{CD} 4^{+}$cell counts of 663.5 (IQR= 570-831) cells $/ \mu \mathrm{L}$ and, at 12 weeks after treatment, had a median $\mathrm{CD}_{4}^{+}$cell count of 418.5 (393-599) cells/ $\mu \mathrm{L}$. For HIV-1 loads, at baseline, the group had a mean $\log _{10}$ HIV-1 plasma viral load of $8.28 \pm 1.45$ (95\% Cl: $7.06-9.49$ ) copies $/ \mathrm{ml}$ and at 12 weeks after treatment, they had a mean $\log _{10} \mathrm{HIV}-1$ plasma viral load of $10.37 \pm$ 1.35 (95\% Cl: 9.24-11.50) copies/ml.

For the study participants who remained with detectable S. mansoni eggs in their stool they had, at baseline, a median CD4 ${ }^{+}$cell count of 472.5 (IQR: $\left.368.5-922\right)$ cells $/ \mu \mathrm{L}$ and, at 12 weeks after treatment, had a median $\mathrm{CD}_{4}{ }^{+}$count of $387(348-426)$ cells $/ \mu \mathrm{L}$. For the HIV-1 viral load, the group had a mean $\log _{10}$ HIV-1 plasma of $9.06 \pm 2.08$ (95\% Cl; 5.74- 12.37) copies/ml and, 12 weeks after treatment, had a mean $\log _{10}$ HIV-1 plasma viral load of $11.46 \pm 0.68$ (95\%Cl; $5.74-12.37$ ) copies $/ \mathrm{ml}$. In general, PZQ treatment did not result in changes of either $\mathrm{CD}_{4}{ }^{+}$cell counts or plasma HIV-1 loads, between baseline and 12 weeks after treatment. No correlation was observed between log S. mansoni egg counts and HIV-1 RNA viral loads ( $r=-0.0689, P=0.77)$ at 6 -month follow-up point in 22 individuals infected with S. mansoni.

\section{Discussion}

The present study highlighted the fact that the six-month follow-up on $\mathrm{CD}^{+}$T-lymphocytes counts did not differ significantly between individuals who were mono-infected with HIV-1 and those who were co-infected with S. mansoni +HIV-1 in a HIV-1 cohort with CD4 $4^{+}$cells counts $\geq 350$ cells $/ \mu \mathrm{L}$. There was a trend in the monthly decrease in $\mathrm{CD} 4^{+}$cell counts with increasing follow-up time points. However, the decrease in $\mathrm{CD}_{4}^{+}$counts did not differ significantly between those mono-infected with HIV-1 and those co-infected with HIV-1+S.mansoni. In relation to plasma HIV-1 loads, at baseline and three-month follow-up, individuals who were identified to be co-infected with HIV-1+S.mansoni at six-month follow-up time point had a significantly higher viral load in comparison to those who were mono-infected with HIV-1. HIV-1 plasma viral loads increased with follow-up time from baseline to six months. Furthermore, praziquantel treatment, evaluated at 12 weeks after treatment, did not results into changes in neither $\log _{10} \mathrm{HIV}-1$ plasma loads nor $\mathrm{CD} 4^{+}$ cell counts in participants who were co-infected with HIV-1+S.mansoni infection. In the present study, we found no correlation between the intensity of S. mansoni infection and $\log _{10} \mathrm{HIV}-1$ plasma viral loads among the co-infected study participants at six-month follow-up time point. 
The relatively small sample size of study participants and a short follow-up time of 6 - 9 months may in part require a cautious interpretation of the findings of the present study. However, these results are notable and, to a degree, support the hypothesis that individuals co-infected with $S$. mansoni and HIV-1 may have significantly higher $\log _{10}$ HIV-1 plasma viral loads.

Our observations on the lack of difference in $\mathrm{CD}_{4}^{+}$cell counts between those who were co-infected with HIV-1+S. mansoni and mono-infected with HIV-1 have also been described in a HIV1 semi-urban cohort in Uganda (Brown et al., 2004). In that cohort, being infected with S. mansoni was not associated with lower $\mathrm{CD}_{4}{ }^{+} \mathrm{T}$-lymphocytes (Brown et al., 2004). Higher and lower $\mathrm{CD} 4^{+}$ cell counts in HIV-1 infected individuals co-infected with different helminths species, including $S$. mansoni, in sub-Saharan Africa have been reported in other studies (Wolday et al., 2002; Brown et al., 2004; Walson \& John-Stewart, 2008). In comparison to other helminth species endemic in subSaharan Africa, in particular gastro-intestinal nematodes, the comparison of $\mathrm{CD}^{+}$cell counts between HIV-1 mono-infected and co-infected individuals at different follow-up time points has revealed no significant difference in lymphocytes counts (Wolday et al., 2002, Walson and JohnStewart, 2008) and profiles (Mkhize-Kwitshana et al., 2011). In the present study, we observed a $\mathrm{CD}_{4}{ }^{+}$cell counts decrease with increased follow-up times for both groups. These observations are expected for individuals infected with HIV-1 and not getting ART, as the pathogenesis of HIV-1 targets the CD4 ${ }^{+}$T-cells (Clerici and Shearer, 1993).

Conversely, comparisons of mean $\log _{10}$ HIV-1 plasma viral loads between individuals coinfected with HIV-1+S.mansoni and those mono-infected with HIV-1 revealed a significant difference in mean viral load at baseline and at three-month follow-up time. However, this difference was not evident at the time of S. mansoni diagnosis at the 6-month time point. As hypothesized elsewhere, chronic immune activation resulting from $S$. mansoni infection may partly explain the difference of mean HIV loads in the two groups (Bentwich et al., 1995; Secor, 2006; Secor et al., 2003; Kamal \& El Sayed Khalifa, 2006). In vitro studies have shown that $\mathrm{Th}_{2}$ cells from S. mansoni infected individuals express a high density of CCR5 and CXCR4 receptors which increases their susceptibility to HIV-1 and HIV-1 replication (Secor et al., 2003). A higher baseline mean HIV-1 viral load has been reported in HIV-1+S. mansoni cohorts in Uganda (Brown et al., 2004); however, this association disappeared after adjusting for other factors such as age, sex and socioeconomic factors, leading to a conclusion that demographic and socio-economic differences are more closely associated with higher HIV-1 viral loads than helminth infections (Brown et al., 2004).

In the present study, after multiple linear regressions analysis, none of the demographic factors (age, sex, village of residence, S. mansoni status and baseline $\mathrm{CD}_{4}^{+}$counts) remained significantly associated with HIV-1 viral loads at baseline. In comparison to other endemic helminths infections in sub-Saharan Africa, such as soil-transmitted helminths, a non-significant difference was observed on mean $\log _{10}$ HIV-1 viral loads among HIV-1+STH co-infected individuals and those mono-infected with HIV-1 in Ethiopia (Wolday et al., 2002). However, in South Africa, HIV-1+STH co-infected individuals who presented with a higher eosinophilia with STH eggs in stool, had almost three-fold higher viral loads and lower absolute $\mathrm{CD} 4^{+}$counts compared to other sub-groups (Mkhize-Kwitshana et al., 2011). Perhaps, there is a variation in the ability of helminths species to activate and modulate the immune system in response to various helminths antigens, which also plays a pivotal role in HIV-1 pathogenesis in sub-Saharan Africa (Mkhize-Kwitshana et al., 2011). When comparing gastrointestinal nematodes and blood trematodes, one expects that S. mansoni, a blood trematode residing in the intravascular system, would locally and systematically activate the immune system more remarkably than the gastro-intestinal nematodes and that, therefore, individuals co-infected with HIV-1+S.mansoni would have higher HIV-1 viral loads than those mono-infected with HIV-1 (Mkhize-Kwitshana et al., 2011).

In the present study, no significant correlation was observed between the intensity of $S$. mansoni infection. A lack of correlation between HIV-1 plasma viral load and changes in S. mansoni burden before and after PZQ treatment have been reported from Western Kenya (Lawn et al., 
2000). Evidence from other helminth species such as STH indicates a strong correlation between the intensity (egg loads) of STH infections and high HIV-1 plasma viral loads (Lawn et al., 2000). In earlier studies in Tanzania (Mazigo et al., 2014) and Zimbabwe (Kallestrup et al., 2005b), no correlation was observed between $\mathrm{CD}_{4}{ }^{+}$cell counts and the intensity of S. mansoni infection.

Based on the immunological evidence on the interactions of HIV-1+S.mansoni in coinfected individuals (Sher \& Coffman, 1992; Secor, 2012), it has been hypothesized that an effective PZQ treatment against S. mansoni in HIV-1 co-infected individuals would result into the reduction of HIV-1 plasma viral loads (Erikstrup et al., 2008) and improve CD4 ${ }^{+}$cell counts. Although the interpretation of our results is limited by a small sample size, PZQ treatment did not result in any change neither in HIV-1 plasma viral loads nor in $\mathrm{CD}_{4}^{+}$cells counts. Similar findings have been reported from Western Kenya (Lawn et al., 2000). In contrast, in rural Zimbabwe, PZQ treatment resulted in the reduction of HIV-1 plasma viral load in the group which received early treatment compared to the group which received a treatment delayed for three months (Kallestrup et al., 2005a).

We observed an insignificant increase in HIV-1 plasma viral loads 12 weeks after treatment, probably indicating that PZQ treatment had no effect on HIV-1 infection. In Uganda (Brown et al., 2005) and Western Kenya (Lawn et al., 2000), an increase in HIV-1 plasma viral loads and decrease in $\mathrm{CD} 4^{+}$cell counts was reported four weeks post PZQ treatment in HIV-1+S.mansoni co-infected individuals. Although the mechanism through which HIV-1 plasma viral loads increase following PZQ treatment remains unknown, there are number of explanations. It is possible that the death of adult schistosomes following treatment results into antigenic stimulations that leads to the activation of the $\mathrm{Th}_{2}$ arm of the immune system and, hence, create a good environment for HIV-1 replication (Joseph et al., 2004; Brown et al., 2005; Chenine et al., 2005; Kamal \& El Sayed Khalifa, 2006). Other authors have suggested that, the effects of S. mansoni infections is highly prolonged and reverses to normal very slowly after praziquantel treatment or eradication of the adult schistosomes compared to gastrointestinal nematodes. This can maintain inflammatory responses important for replication of HIV-1. Alternatively, praziquantel treatment results into the suppression of the production of inflammatory and anti-inflammatory cytokines that are maintained by chronic helminth infections (Joseph et al., 2004). In part, the increase of HIV-1 viral loads following praziquantel treatment is thought to result into a decline in T-cell activities which in turn suppress antiviral immune responses (Aandahl et al., 2004).

Our study was subjected to some limitations; it was not possible to determine the exact time the study participants were either infected with HIV-1 or S. mansoni. Similarly, the use of one Kato Katz technique, to characterized individuals infected or not infected with S. mansoni based on egg counts could have resulted into a false negative misclassification of individuals. However, studies using more sensitive techniques, such as circulating cathodic antigen test (CCA) or Circulating Anodic Antigen (CAA) test have produced similar results (Lawn et al., 2000). The small sample size also limited the study. It is worthwhile to note that, it was not possible to measure humoral responses (antibodies responses) and cytokines to verify some of the observations. These factors may have confounded the study. Nevertheless, this was a community prospective longitudinal survey and the results are notable.

In conclusion, we found that, mean HIV-1 plasma viral loads varied significantly, with coinfected individuals having a higher mean at baseline and at three-month follow up time point than the HIV-1 mono-infected individuals. Furthermore, no correlation was observed between mean HIV-1 plasma viral loads and the intensity of S. mansoni infection. The treatment of $S$. mansoni infection in co-infected individuals did not result in changes in either HIV-1 plasma viral loads or $\mathrm{CD}_{4}^{+}$cell counts. However, based on the available evidence on the immunological interaction of HIV-1+S.mansoni infections, our results do not disqualify the bio-directional effects of each infection on their pathogenesis in co-infected individuals. In addition, although deworming may not have effects on HIV-1 markers in co-infected individuals, at least for the present study population, it should be part of the HIV-1 care to reduce and control the co- 
morbidities associated with S. mansoni infection in co-infected individuals. Further studies are recommended to understand, in a larger cohort, the biological interactions of the two infections and the effects of deworming on the risk of HIV-1 transmission and its progression in sub-Saharan Africa.

\section{Author's contributions}

HDM, DWD, SMK, FN designed the study. HDM, DM and FN participated in data analysis. HDM drafted the first version of the manuscript. All authors read and approved the final manuscript, contributed to the critical review and made substantial contributions to its content.

\section{Acknowledgements}

This work was supported by Training Health Researchers into Vocational Excellence in East Africa (THRiVE), grant number 087540 funded by the Wellcome Trust. Its contents are solely the responsibility of the authors and do not necessarily represent the official views of the supporting offices. DWD and HDM acknowledge additional funds from Cambridge-Africa Alborada Research fund. HDM acknowledge additional funding support from Dan Davis Prize Scholarship, Tel Aviv University, Israel and the Special Programme for Research and Training in Tropical Diseases. We acknowledge the technical and financial assistance from the National Institute for Medical Research in Mwanza and the Catholic University of Health and Allied Sciences and Bugando Medical Centre, Mwanza. We thank all the study participants and the local government for allowing us to carry out this study.

\section{References}

Aandahl, E.M., Michaelsson, J., Moretto, W., Hecht, F.M. \& Nixon, D.F. (2004) Human CD4+ CD25+ regulatory cells control $\mathrm{T}$-cell response to human immunodeficiency virus and cytomegalovirus antigens. . Journal of Virology 78, 2454 - 2459.

Bentwich, Z., Kalinkovich, A. \& Weisman, Z. (1995) Immune activation is a dominant factor in the pathogenesis of African AIDS. Immunollogy Today 16, 187-191.

Bentwich, Z., Kalinkovich, A., Weisman, Z., Borkow, G., Beyers, N. \& Beyers, A.D. (1999) Can eradication of helminthic infections change the face of AIDS and tuberculosis? Immunollogy Today 20, 485-487.

Bentwich, Z., Maartens, G., Torten, D., Lal, A. A. \& Lal, R. B. (2000) Concurrent infections and HIV pathogenesis. AIDS 14, 2071-2081.

Borato, D.C.K., Carraro, E., Ribas, S.R.W., Kalva-Filho, C.A. \& Vellosa, J.C.R. (2012) Comparison of Two Methodologies for CD4+ T Lymphocytes Relative Counting on Immune Monitoring of Patients with Human Immunodeficiency Virus. The Science World Journal Volume? pages?????

Borkow G \& Bentwich, Z. (2006) HIV and helminth co-infection:is deworming necessary? Parasites Immunology 28, 605 - 612 .

Borkow, G. \& Bentwich, Z. (2004) Chronic immune activation associated with chronic helminthic and human immunodeficiency virus infections: role of hyporesponsiveness and anergy. Clinical Microbiology Review 17, 1012-1030.

Brown, M., Kizza, M., Watera, C., Quigley, M.A., Rowland, S., Hughes, P., Whitworth, J. A. \& Elliott, A. M. (2004) Helminth infection is not associated with faster progression of HIV disease in coinfected adults in Uganda. Journal of Infectious Diseases 190, 1869-1879.

Brown, M., Mawa, P. A., Joseph, S., Bukusuba, J., Watera, C., Whitworth, J. A., Dunne, D. W. \& Elliott, A. M. (2005) Treatment of Schistosoma mansoni infection increases helminthspecific type 2 cytokine responses and HIV-1 loads in coinfected Ugandan adults. Journal of Infectious Diseases 191, 1648-1657. 
Chenine, A.L., Buckley, K.A., Li, P.L., Rasmussen, R.A., Ong, H., Jiang, S., Wang, T., Augostini, P., Secor, W.E. \& Ruprecht, R.M. (2005) Schistosoma mansoni infection promotes SHIV clade $C$ replication in rhesus macaques. AIDS 19, 1793-1797.

Clerici, M. \& Shearer, G. M. (1993) A TH1-->TH2 switch is a critical step in the etiology of HIV infection. Immunollogy Today 14, 107-111.

Erikstrup, C., Kallestrup, P., Zinyama-Gutsire, R.B.L., Gomo, E., Van Dam, G.J., Deelder, A. M., Butterworth, A.E., Pedersen, B.K., Ostrowski, S.R., Gerstoft, J. \& Ullum, H. (2008) Schistosomiasis and infection with Human Immunodeficiency Virus 1 in rural Zimbabwe: Systematic inflammatory during co-infection and after treatment for schistosomiasis. American Journal of Tropical Medicine and Hygiene 79, 331-337.

Joseph, S., Jones, F.M., Walter, K., Fulford, A.J., Kimani, G., Mwatha, J.K., Kamau, T., Kariuki, H.C., Kazibwe, F., Tukahebwa, E., Kabatereine, N.B., Ouma, J.H., Vennervald, B.J. \& Dunne, D.W. (2004) Increases in human T helper 2 cytokine responses to Schistosoma mansoni worm and worm-tegument antigens are induced by treatment with praziquantel. Journal of Infectious Diseases, 190, 835-842.

Kalinkovich, A., Borkow, G., Weisman, Z., Tsimanis, A., Stein, M. \& Bentwich, Z. (2001) Increased CCR5 and CXCR4 expression in Ethiopians living in Israel: environmental and constitutive factors. Clinical Immunology 100, 107-117.

Kalinkovich, A., Weisman, Z., Greenberg, Z., Nahmias, J., Eitan, S., Stein, M. \& Bentwich, Z. (1998) Decreased CD4 and increased CD8 counts with $T$ cell activation is associated with chronic helminth infection. Clinical Exprimental Immunology 114, 414-121.

Kallestrup, P., Zinyama, R., Gomo, E., Butterworth, A. E., Mudenge, B., van Dam, G. J., Gerstoft, J., Erikstrup, C. \& Ullum, H. (2005a) Schistosomiasis and HIV-1 infection in rural Zimbabwe: effect of treatment of schistosomiasis on CD4 cell count and plasma HIV-1 RNA load. Journal of Infectious Diseases, 192, 1956-1961.

Kallestrup, P., Zinyama, R., Gomo, E., Butterworth, A. E., van Dam, G. J., Erikstrup, C. \& Ullum, H. (2005b) Schistosomiasis and HIV-1 infection in rural Zimbabwe: implications of coinfection for excretion of eggs. Journal of Infectious Diseases 191, 1311-1320.

Kamal, S.M. \& El Sayed Khalifa, K. (2006) Immune modulation by helminthic infections: worms and viral infections. Parasite Immunology 28, 483-496.

Katz, N., Chaves, A. \& Pellegrino, J. (1972) A simple device for quantitative stool thick-smear technique in Schistosomiasis mansoni. Revista do Instituto de Medicina Tropical de Sao Paulo, 14, 397-400.

Lawn, S.D., Karanja, D.M., Mwinzia, P., Andove, J., Colley, D.G., Folks, T.M. \& Secor, W.E. (2000) The effect of treatment of schistosomiasis on blood plasma HIV-1 RNA concentration in coinfected individuals. AIDS 14, 2437-2443.

Lyamuya, E.F., Aboud, S., Urassa, W.K., Sufi, J., Mbwana, J., Ndugulile, F. \& Massambu, C. (2009) Evaluation of simple rapid HIV assays and development of national rapid HIV test algorithms in Dar es Salaam, Tanzania. BMC Infectious Diseases 9, 19.

Maizels, R.M., Bundy, D.A., Selkirk, M.E., Smith, D.F. \& Anderson, R.M. (1993) Immunological modulation and evasion by helminth parasites in human populations. Nature 365, 797805.

Maizels, R.M. \& Yazdanbakhsh, M. (2003) Immune regulation by helminth parasites: cellular and molecular mechanisms. Nature Review: Immunology, 3, 733-44.

Mazigo, H.D., Dunne, D.W., Kinung'hi, S.M. \& Nuwaha, F. (2014a) Praziquantel efficacy against Schistosoma mansoni among HIV-1 infected and uninfected adults living in fishing villages along Lake Victoria, Northwest Tanzania. Infectious Disease of Poverty 3, 47.

Mazigo, H.D., Dunne, D.W., Wilson, S., Kinung'hi, S.M., de Moira, A., Jones, F.M., Morona, D. \& Nuwaha, F. (2014b) Co-infection with Schistosoma mansoni and Human Immunodeficiency Virus-1 (HIV-1) among residents of fishing villages of north-western Tanzania. Parasites \& Vectors $7,587$. 
Mazigo, H.D., Nuwaha, F., Wilson, S., Kinung'hi, S.M., Morona, D., Waihenya, R., Heukelbach, J. \& Dunne, D.W. (2013) Epidemiology and interactions of Human Immunodeficiency Virus - 1 and Schistosoma mansoni in sub-Saharan Africa. Infectious Diseases of Poverty 2, 2.

Mkhize-Kwitshana, Z.L., Taylor, M., Jooste, P., Mabaso, M.L. \& Walzl, G. (2011) The influence of different helminth infection phenotypes on immune responses against HIV in co-infected adults in South Africa. BMC Infectious Diseases 11, 273.

Modjarrad, K. \& Vermund, S.H. (2010). Effect of treating co-infections on HIV-1 viral load: a systematic review. Lancet Infectious Diseases 10, 455-463.

Mwinzi, P.N., Karanja, D.M., Colley, D.G., Orago, A.S. \& Secor, W.E. (2001) Cellular immune responses of schistosomiasis patients are altered by human immunodeficiency virus type 1 coinfection. Journal of Infectious Disease 184, 488-496.

Secor, W.E. (2005) Immunology of human schistosomiasis: off the beaten path. Parasite Immunology 27, 309-316.

Secor, W.E. (2006) Interactions between schistosomiasis and infection with HIV-1. Parasite Immunology 28, 597-603.

Secor, W.E. (2012) The effects of schistosomiasis on HIV/AIDS infection, progression and transmission. Current Opinion in HIV AIDS 7, 254-259.

Secor, W.E., Karanja, D.M.S. \& Colley, D.G. (2004) Interactions between schistosomiasis and Human Immunodeficiency Virus in Western Kenya. Memerios do Instistuto Oswaldo Cruz Rio de Janeiro 99, 93-95.

Secor, W.E., Shah, A., Mwinzi, P.M., Ndenga, B.A., Watta, C.O. \& Karanja, D.M. (2003) Increased density of human immunodeficiency virus type 1 coreceptors CCR5 and CXCR4 on the surfaces of CD4(+) T cells and monocytes of patients with Schistosoma mansoni infection. Infection and Immunity 71, 6668-6671.

Sher, A., Gazzinelli, R., Oswald, I.P., Clerici, M., Kullberg, M., Pearce, E.J., Berzofsky, J.A., Mosmann, T.R., James, S.L. \& Morse, H.C. (1992) Role of T-cell derived cytokines in the downregulation of immune responses in parasitic and retroviral infections. Immunology Review 127, 183 - 204.

Sher, A.F. \& Coffman, R.L. (1992) Regulation of immunity to parasites and the hygiene hypothesis. Science 296, 372-377.

Walson, J.L., Otieno, P.A., Mbuchi, M., Richardson, B.A., Lohman-Payne, B., Macharia, S.W., Overbaugh, J., Berkeley, J., Sanders, E.J., Chung, M.H. \& John-Stewart, G.C. (2008) Albendazole treatment of HIV-1 and helminth co-infection: a randomized,double-blind, placebo-controlled trials. AIDS 22, 1601-1609.

Walson, J.L. \& John-Stewart, G. (2007) Treatment of helminth co-infection in individuals with HIV1: A systematic review of the literature. PLoS Neglected Tropical Diseases 1, e102.

Walson, J. L. \& John-Stewart, G. (2008) Treatment of helminth co-infection in HIV-1 infected individuals in resource-limited settings. Cochrane Database Systematic Review, CDoo6419.

Wolday, D., Mayaan, S., Mariam, Z. G., Berhe, N., Seboxa, T., Britton, S., Galai, N., Landay, A. \& Bentwich, Z. (2002) Treatment of intestinal worms is associated with decreased HIV plasma viral load. Journal of Acquired Immune Deficiency Syndrome 31, 56-62. 